\title{
Type 2 diabetes structured education provision in Yorkshire and the Humber 2014/15
}

\author{
MATT GREENSMITH, HELEN ATKINSON, BRYAN POWER, CHRIS WALTON, YORKSHIRE AND THE \\ HUMBER DIABETES CLINICAL NETWORK
}

\begin{abstract}
The Clinical Commissioning Group (CCG) Improvement and Assessment Framework published in June 2016 places a focus on diabetes patient education with a metric for 'people with diabetes diagnosed less than a year who attend a structured education course'. Despite the importance of structured education for people newly diagnosed with diabetes, data around service activity is poor. The Diabetes Clinical Network in Yorkshire and the Humber sought to obtain a clear picture of structured education provision and services to inform commissioners and providers across the region. This report summarises a data collection exercise for the time period April 2014 to March 2015. In 2014/2015, 11 CCGs had type 2 structured education attendance of $30 \%$ or greater. The results were far higher than the $5.9 \%$ average for Yorkshire and the Humber in the National Diabetes Audit (NDA). In 2014/2015, attendance at structured patient education programmes in the region was on average nearly 5 times higher than reported in the NDA. Whilst the patient education attendance data from providers are significantly better than those reported nationally, they are far lower than is desirable with, on average, over $70 \%$ of newly diagnosed patients not attending.
\end{abstract}

Br J Diabetes 2017;17:14-16

Key words: diabetes, structured patient education, CCG Improvement and Assessment Framework

\section{Introduction}

There has been a shift in national policy in recent years with greater emphasis on services that enable 'self-care' for patients with longterm conditions. ${ }^{1}$ National Institute of Health and Care Excellence

North Region (Yorkshire and the Humber), NHS England, Willerby, East Yorkshire, UK

Address for correspondence: Mr Matt Greensmith Quality Improvement Manager (Diabetes), Clinical Networks, North Region (Yorkshire and the Humber), NHS England, Health House, Grange Park Lane, Willerby, East Yorkshire HU10 6DT, UK E-mail: matt.greensmith@nhs.net

http://dx.doi.org/10.15277/bjd.2017.115
(NICE) guidance recommends that structured patient education should be offered to adults with type 2 diabetes and/or their family members or carers (as appropriate) at and around the time of diagnosis, with annual reinforcement and review. ${ }^{2}$

The increased prominence of diabetes structured education in national policy is evidenced by its selection as one of only two diabetes metrics (alongside attainment of NICE treatment targets) in the recently published CCG Improvement and Assessment Framework (CCGIAF) 'People with diabetes diagnosed less than a year who attend a structured education course', ${ }^{3}$ and remains as a metric in the ongoing CCG outcome indicator set 'a person is referred for structured education within 12 months of being diagnosed with diabetes'. ${ }^{4}$

Structured education programmes for people newly diagnosed with diabetes are a key component of effective self-care and diabetes management. However, despite the importance of structured education, data around service activity is poor.

Existing sources of information on structured education do not provide a complete picture of education provision. The Quality and Outcomes Framework (QOF) incentivises the recording of referrals from primary care into structured education (QOF ID DM014), ${ }^{5}$ although there is no incentive within QOF to record attendance from the provider in the primary care record. This is important as it is this recording of attendance in general practice patient records that is extracted and reported by the NDA. ${ }^{6}$ The reported attendance data from the NDA audit remains very low and is considered to under-represent actual attendance.

To better understand the provision and uptake of structured patient education for people with type 2 diabetes in the region, the Yorkshire and Humber Diabetes Clinical Network undertook a survey and data collection exercise with providers of structured patient education for 2014/2015.7

\section{Methods}

The Yorkshire and Humber Clinical Networks undertook a review of service provision and uptake across Yorkshire and the Humber. Education providers were asked to report on the services provided from April 2014 to March 2015 by the CCG. Providers were identified and contacted by telephone and were asked a series of qualitative questions relating to their service and its provision. A short data return template was distributed to education providers to collect additional quantitative data relating to refer- 
Figure 1. NDA reported attendance figures against the provider-reported figure, 2014/2015. Dark blue bars show the NDA number attended as a percentage of NDA newly diagnosed and light blue bars show the provider number attended as a percentage of QOF newly diagnosed

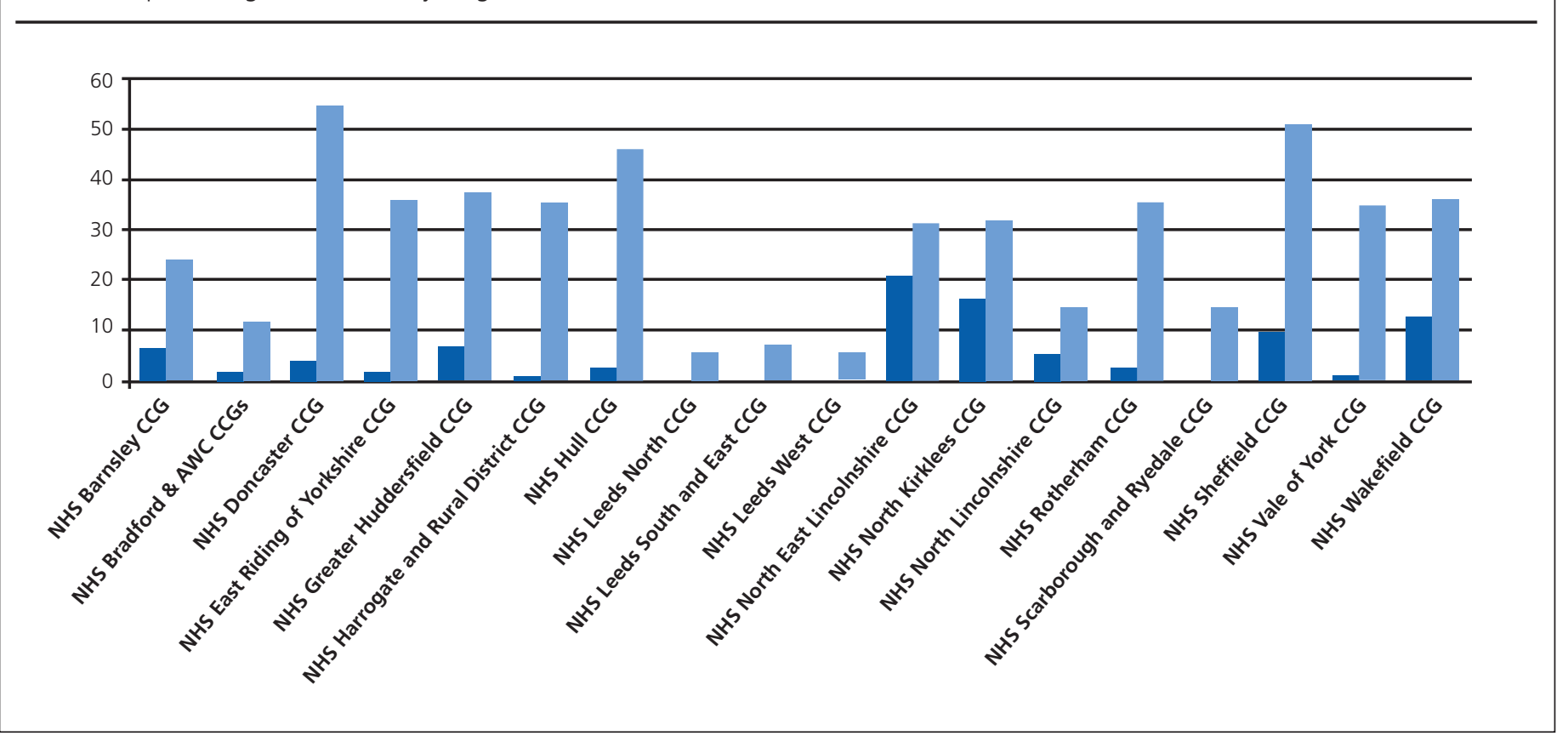

ral, attendance at and completion of structured education. All providers were offered an opportunity to check and revise their submitted data before and after seeing a draft of the final report.

\section{Results}

Structured education data for type 2 diabetes reported by the NDA significantly under-represents attendance for structured patient education in Yorkshire and the Humber (Figure 1). In 2014/2015 the provider reported attendance figures for the 23 Yorkshire and the Humber CCGs equating to an average of $28 \%$ of QOF newly diagnosed patients compared with the NDA attendance figure of an average of $5.9 \%$ of NDA newly diagnosed patients in Yorkshire and the Humber. Using the clinical network methodology to calculate attendance in 2014/2015, 11 CCGs had an attendance of $30 \%$ or greater.

Whilst the patient education attendance data from providers are significantly better than those reported nationally, they are far lower than is desirable with on average over $70 \%$ of newly diagnosed patients not attending.

\section{Discussion}

Because the NDA extracts structured education attendance data from primary care clinical systems, it is likely that the under-reporting of attendance is in large part due to deficiencies in the communication and coding of attendance between education providers and primary care clinical systems. In order to obtain accurate information on the uptake of structured education, data can be obtained directly from providers. Commissioners and providers should work together to identify and specify a common dataset and approaches to coding that enable accuracy in the recording of attendance. The impact of efforts to increase patient attendance will be difficult to measure under the current coding and recording arrangements (current at time of completion of report in June 2016).

\section{Limitations of study}

This exercise only collected data on education provision for patients with type 2 diabetes. Type 1 education was excluded from this process.

The timescale for calculating attendance differed between the NDA and the Clinical Network exercise. The NDA looks at certain clinical codes for attendance over a 15-month period using the NDA reported percentage for newly diagnosed patients as a denominator. To gain a more complete picture and to take into account low participation rates in the NDA, the Clinical Network exercise used provider-reported attendance data for a 12-month period using QOF data for patients newly diagnosed with diabetes within the same period as a denominator.

Some providers offer structured education programmes to patients from more than one CCG, and they were asked to return data at the CCG level. One provider was not able to split their data and so provided combined aggregate data for all of their CCGs. In some cases, the providers were not able to distinguish between patients who had received the full course and those who had only attended part of an education course.

This was the first time that education providers had been asked to collect and report these data, and this presented a number of data recording and data reporting-related issues including:

a. a change in the system used to record activity across the audit period; 


\section{Key messages}

- Attendance at structured education is significantly higher than suggested in NDA data

- However, on average over $70 \%$ of newly diagnosed patients still do not attend

- In most cases, providers of structured education can provide accurate data on attendance to inform local service improvement initiatives

b. long waiting times for data extraction;

c. difficulty in establishing the parameters of the search;

d. a change in course and course length during the audit period;

e. identification of newly diagnosed patients.

\section{Conclusion}

As with any first data return, the results should be treated with a degree of caution. Further work should be carried out to understand why up to $70 \%$ of newly diagnosed patients that are invited do not attend. Providers and commissioners should work with local populations to identify barriers to attendance and consider how patient insight can be included in designing structured education programmes.

\section{Conflict of interest None. Funding None}

\section{References}

1. https://www.england.nhs.uk/wp-content/uploads/2014/10/5yfv-web.pdf

2. https://www.nice.org.uk/guidance/ng28/chapter/Key-priorities-for-implementation

3. https://www.england.nhs.uk/commissioning/wp-content/uploads/sites/ 12/2016/03/ccg-iaf-mar16.pdf

4. https://www.england.nhs.uk/resources/resources-for-ccgs/ccg-outtool/ccg-ois/

5. http://www.nhsemployers.org/your-workforce/primary-care-contacts/ general-medical-services/quality-and-outcomes-framework

6. http://www.hscic.gov.uk/nda

7. http://www.yhscn.nhs.uk/cardiovascular/Diabetes/SPE.php

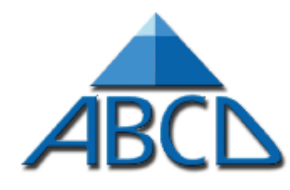

Association of British Clinical Diabetologists

\title{
Empagliflozin (Jardiance) Nationwide Audit in progress!
}

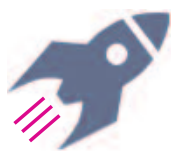

$A B C D$ has launched a nationwide audit of Empagliflozin in the UK to assess real clinical efficacy and safety \& inform future practice and guidelines

\section{Does your centre use Empagliflozin (Jardiance)?}

\author{
If yes, REGISTER YOUR CENTRE at
}

http://www.diabetologists-abcd.org.uk/n3/Empagliflozin_Audit.htm

- you are invited to enter your patients' data into the bespoke online tool

- you are able to analyse your local data easily

- the data will be automatically added to the national data in anonymised form

- we can provide easy-to-complete paper proformas for use in clinic if preferred

\section{Please remember:}

- the more data, the more complete our understanding of Empagliflozin in real clinical practice

- all contributors will be listed in publications arising from data submission 